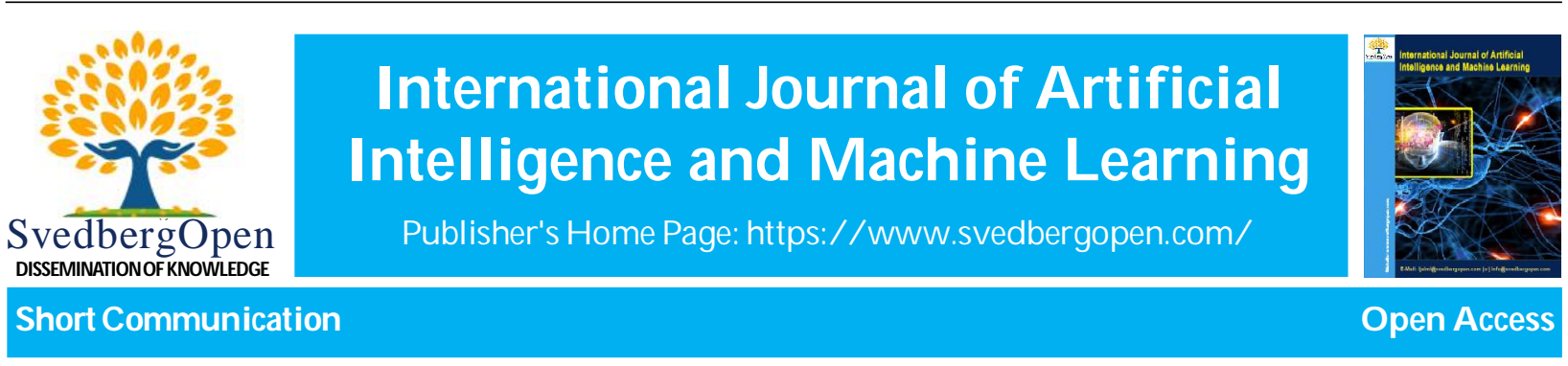

\title{
How decentralized systems can upgrade AI
}

\author{
Wulf A. Kaal ${ }^{*}$ \\ ${ }^{1}$ Professor, Director of the Private Investment Fund Institute, University of St. Thomas School of Law, 1000 LaSalle Avenue, MSL \\ 400 Minneapolis, MN 55403. E-mail: wulfkaal@stthomas.edu
}

\section{Article Info}

Volume 1, Issue 1, July 2021

Received : 24 February 2021

Accepted : 17 June 2021

Published : 05 July 2021

doi: 10.51483/IJAIML.1.1.2021.1-10

\begin{abstract}
Decentralized systems enhance future algorithmic applications. Centralized algorithmic automation is a very powerful tool for humanity. It fulfills increasingly important roles in society for the betterment of humanity. It optimizes human interaction, outputs, and performance and can elevate most functions of technology-enhanced designs in society. At the same time, the conveniences and benefits that derive from centralized algorithmic automation bring with them risks to humanity that cannot be fully quantified. Decentralized systems can help counteract the downsides and threats of algorithmic automation and thereby enhance the long-term benefits of technological solutions for humanity.

Keywords: Decentralization, AI, Algorithm, Digital assets, Decentralized Finance, Blockchain, Decentralized commerce, Incentive design, Tokens, Decentralized Infrastructure

(C) 2021 Wulf A Kaal. This is an open access article under the CC BY license (https://creativecommons.org/licenses/by/4.0/), which permits unrestricted use, distribution, and reproduction in any medium, provided you give appropriate credit to the original author(s) and the source, provide a link to the Creative Commons license, and indicate if changes were made.
\end{abstract}

\section{Introduction}

\subsection{Technology-Enhanced Individualism}

Decentralized systems constitute a form of technology-enhanced individualism. Individualism in this context means the political philosophy that emphasizes the moral worth of individuals and their importance over the state and social groups. ${ }^{1}$ Similarly, liberalism, anarchism, and existentialism focus on the human individual in their analysis of society. ${ }^{2}$ Whereas these political philosophies focused on the human individual's role in society with a strong emphasis on personal freedom, decentralized systems focus more on the role of the individual and the individual's unique contributions to the collective reasoning process that then advances or protects society. Individual freedom is attained indirectly through the decentralized betterment of the system and society. Decentralized systems enable a feedback mechanism between unique individualist inputs and personal individual freedoms. As the system improves with the quality of

\footnotetext{
* Corresponding author: Wulf A. Kaal, Professor, Director of the Private Investment Fund Institute, University of St. Thomas School of Law, 1000 LaSalle Avenue, MSL 400 Minneapolis, MN 55403. E-mail: wulfkaal@stthomas.edu

1 "The emancipation of the individual was indeed the great spiritual revolution which had led to the breakdown of tribalism and to the rise of democracy. (...) This individualism, united with altruism, has become the basis of our western civilization. (...) There is no other thought which has been so powerful in the moral development of man." See Chapter 2, FN 23 [Popper, Chapter 6].

2 Wikipedia, Individualism, https://en.wikipedia.org/wiki/Individualism (accessed on June 1, 2020).
} 
unique individual inputs, the system increasingly values the uniqueness of human inputs, and as a result, personal liberties and freedom increase.

Features of collectivism in decentralized systems can be distinguished from collectivism as a political philosophy. Collectivism is defined as the practice or principle of giving a group priority over the individuals in such group. In a perfect world, decentralized systems recognize human individuals as ends in themselves. ${ }^{3}$ In this sense, decentralized systems may be contrasted with collectivism that sees individuals as means to an end. Where collectivism emphasizes the cohesiveness among individuals with prioritization of the group over the self, ${ }^{4}$ decentralized computing systems emphasize the wisdom of the crowds through the unique individual inputs of the members of the crowd. Decentralized computing systems and networks typically intend prioritize network effects and the collective wisdom of the group for the betterment of society. Where collectivism creates limitations on individuals or demands sacrifices from individuals for the benefit of the group, decentralized computing networks emphasize the importance of the individual in the collective engagement that in effect improves outcomes for the group. Where collectivism would demand a sacrifice of personal fulfilment for the group, decentralized systems would prioritize personal fulfilment as it enhances unique inputs and innovation that increase the collective wisdom of the group.

Decentralized technology enhances critical rationalism, individualism, and the growth of human reason. Decentralization can protect humanity from the corruptive influences of ethical collectivism. Both liberalism and collectivism appeal to the emotionally satisfying common purpose as a means of enhancing a collective outcome for society. Appeals to a human individualistic sacrifice for a common good is a form of ethical collectivism which corrupts society and undermines reason and rational thoughts of individuals. ${ }^{5}$

\section{Decentralism transcends capitalism and socialism}

In the $20^{\text {th }}$ and $21^{\text {st }}$ centuries, human society and economic systems have revolved around and evolved from political and economic doctrines that seek to organize society effectively and allocate resources efficiently for the betterment of humanity. First and foremost among those are the dichotomies between capitalism and socialism. Society is organized around those notions and has formed power structures and political hierarchies to serve their respective underlying ideas.

Differences between socialism and capitalism manifest themselves in almost all metrics of societal organization. Very few societies are exclusively run based on either capitalistic or socialistic doctrine. Rather, policy in a given society is an amalgamation of capitalistic ideas and socialistic ideas. The degree of capitalistic ideas versus socialistic ideas in a given society materialize through its adherence to basic metrics. Such metrics may include notions of ownership, equality, the role of government, markets, prices, efficiency, taxes, and healthcare, among many others. Where capitalism sees ownership as assets that are owned by private individuals and firms, socialism suggests assets should be owned by co-operatives and by the government. A core difference between socialism and capitalism revolves around efficiency. Whereas capitalism suggest that market incentives encourage firms to cut costs and increase efficiency of operations, socialism is less focused on notions of efficiency as government owned firms have fewer incentives to increase efficiencies that create economies of scale. Where capitalism sees equality created by markets and income determined by market forces, socialism holds that private income should be redistributed by a strong government to ensure equality of its citizens. Similarly, taxes in capitalism are limited to limit government spending, whereas socialist systems typically have high progressive taxes combined with higher spending on public services. Prices in capitalism are determined by market supply and demand, whereas prices are subject to governmental price controls in socialism.

The long-lasting debate on benefits and detriments of capitalism and socialism is largely pointless. Capitalism has advantages through a dynamic economy and strong incentives for innovation and economic growth. By contrast, socialism has significant strengths in the context of the promotion of equality and its attempts to overcome market failure. Negative outcomes of capitalism and socialism are equally bountiful. Capitalism can lead to significant inequities, inequality, as well as monopolies, and other market failures. Similarly, socialism has significant incentive design issues, where hard work is not rewarded effectively and external costs are largely ignored. More socialist systems are afflicted with significant inefficiencies of state-run industries.

\footnotetext{
3 This is an analogy to Kant's central practical doctrine which recognizes that human individuals are ends, and not to be used as means to an end. Kant, Immanuel (1785). Grundlegung zur Metaphysik der Sitten [German]. Riga, J.F. Hartknoch.

4 Wikipedia, Collectivism, https://en.wikipedia.org/wiki/Collectivism (accessed June 1, 2020).

5 "If the growth of reason is to continue, and human rationality to survive, then the diversity of individuals and their opinion, aims, and purposes must never be interfered with... . Even the emotionally satisfying appeal for a common purpose, however, excellent, is an appeal to abandon all rival moral opinions and the cross-criticisms and arguments to which they give rise. It is an appeal to abandon rational thought." Karl Popper 'The Poverty of Historicism'; "What I assert is that this moral principle which posits 'the general good of society' as a moral aim, is not good enough as a basis of ethics; for example, that it leads to lying - 'for the general good of society' or 'for the benefit of the city'. In other words, I try to show that ethical collectivism is mischievous, that that it corrupts. See Chapter 2, FN 23 [Popper].
} 
Decentralism transcends socialism and capitalism. Decentralism posits that centralized coordination of societal and economic activity, as instituted and instantiated in capitalistic or socialistic doctrine, should be held to a minimum in order to free the human potential. Decentralism constitutes a form of technology-enhanced individualism that builds on and reforms society's existing infrastructure. Where the dichotomies between capitalism and socialism focus on ownership as assets that are owned either private individuals and firms (capitalism) or by co-operatives and by the government (socialism), decentralism focuses on the largest possible utility of assets through personal ownership or collective ownership for human kind. Where the dichotomies between capitalism and socialism focus on efficiency, that is, whether market incentives encourage firms to cut costs and increase efficiency of operations (capitalism) or the government owned firms have fewer incentives to increase efficiencies that create economies of scale (socialism), decentralism creates efficiency not in linear forms but via higher levels of connectivity. Where capitalism and socialism see equality created by markets and income determined by market forces (capitalism) or holds that private income should be redistributed by a strong government to ensure equality of its citizens (socialism), decentralism creates higher levels of equality in society through the preservation of diversity of content, diversity of data sources, and enhanced node connectivity. Where the dichotomies between capitalism and socialism debate equitable redistribution by either curtailing taxes to limit government spending and economic growth (capitalism) or by increasing progressive taxes combined with higher spending on public services (socialism), decentralism's redistribution is based on ownership rights in persons' individuality-derived data that creates economic value. Where capitalism is focused on economies of scale, maximum production, and economic growth, socialism is often seen as the antithesis with more focus on long-term sustainable economic solutions through planning economies. By contrast, decentralism is less concerned with economic growth through per unit cost reduction, economies of scale, and the associated negative externalities, but rather is focused on connectivity and growth through network effects. As such, negative externalities are minimized without centralized planning.

The transcendental nature of decentralism is manifested through the instantiation of its core features in different systems and contexts. For example, technology is largely limited to the control of corporations (capitalism) or governments (socialism). By contrast, in decentralism, technologies are mostly shared to create best solutions for humanity and not owned and controlled. The open source movement, in its purest form, provides an example of the power and use of technology for the greatest utility of humanity. Similarly, the arts and sciences are subject of the limitations of monetary quantification in both capitalism and socialism. By contrast, because the arts and science help proliferate unique skillsets and diversity of opinions, decentralism values the arts and sciences disproportionately. Human rights, access to resources, sustainability, freedom, peace and prosperity are also protected at different levels in capitalistic or socialistic societies. The highest protection of human rights, access to resources, sustainability, freedom, peace and prosperity around the globe is universally emphasized in the technology-enhanced individualism of decentralism as each ensures the foundations of human input into decentralized systems.

As compared with capitalism and socialism, the role of trust is different in decentralism. In capitalism, trust in the system is generated through trusted intermediaries and checks and balances. Trust in socialism is created through belief in the infallibility of government. By contrast, decentralism posits that trust in the system should not be controlled by corruptible human constructs and institutions that are controlled by a limited number of fallible and corruptible humans. Rather, trust in decentralism is based on technology-enhanced individualism. Trust depends on technology-facilitated collective human action and as such belongs to the majority, incorruptible by fallible individual action. Trust in capitalism and socialism is partially created by legal guarantees and by a judicial system that enforces such legal guarantees. Legally created trust is often limited as it can be arbitrary because it is only indirectly democratically legitimized, inflexible, untimely, resistant to change, with fallible human-centric decision processes, and human speed. Trust in decentralized legal solutions can be more democratically legitimized through microdemocratic, individualistic yet technology-centric autonomous decision processes, coded guarantees, and machine speed.

Decentralism allows society to reevaluate its forms of organization and production. Decentralized systems use elements of profit generation and redistribution in a way that in effect combines capitalistic and socialistic ideas. Decentralism allows for the organization of society in economic structures that generate profits while at the same time redistributing resources. For example, DAOs combine elements of socialistic cooperatives with the meritocracy and incentivization of capitalism. Another example is provided by the ability to sell one's personal (e.g., social media etc. consumption) data, preferences, opinions etc. in decentralized systems, including in real time. Such assets, e.g., one's data, preferences, opinions etc., can only limitedly be commercialized in existing centralized structures. In decentralized structures such assets can be tokenized, valued, and mobilized. Similarly, tokenized hard assets, such as real-estate, can be tokenized and sold in unprecedented fractional forms. The ability to control one's data and fractionalized (hard) 
assets enables new forms of consumption for consumers. In early 2020, several startups were developing a barter system in which services, such as free car rides etc., become available in return for disclosure of consumers' data and personal preferences etc.

\section{Technological microdemocracy}

"The printing press taught the people how to read; the internet taught the people how to write; decentralized technology taught the people how to vote." ${ }^{6}$

Representative democracies in the $21^{\text {st }}$ century are subject to significant shortcomings. The very notion of delegating one's vote to a third party without true knowledge of the ethics and belief system of such a third party creates suboptimal outcomes. Delegated voting on issues one cannot foresee corrupts. Voted representatives are not incentivized to vote for outcomes that reflect the presumptive wishes and needs of their constituents but for maintaining their own power of office. Voting incentives and outcomes are different in direct democratic votes. In ancient Greece, citizens voted on any public issues directly on the town's square. Public issues that citizens engaged on in those days could be as mundane as where to build the next road. Incentives, voting outcomes, and democratic legitimacy were aligned in those days. Democracy is increasingly under threat and needs upgrades.

Historically, it was not physically or technologically possible for all constituents in a given democratically organized institution or state to convene physically or digitally to vote on societal and public matters. To overcome these physical, practical, and technological shortcomings, democratic societies devised a system of democratic representation where only a limited number of elected representatives makes decisions on behalf of their constituents. Because of the technological, physical, and practical limitations faced by early democracies, the downsides of democratic representations, such as corruption, bribery, and biases, had to be tolerated as inevitable and necessary tradeoffs. The nature of delegating authority to representatives in combination with boundedly rational human nature and lacking incentivization for ethical conduct necessitated an external ex-post monitoring system that attempts to remedy bribery, lobbying, bias, coverups, and corruption. Ex ante remedies to overcome these downsides of representative democracies were not technologically possible.

Microdemocratic principles can help overcome the existing challenges of representative democracies. Microdemocratic applications embrace technology to eliminate many of the problems faced by representative democracies. For example, internet-based technology and proper technological incentivization can increase lacking voter participation that undermines representative democracies. Experimentation with electronic forms of voting allows new forms of allocating votes to prioritize societal issues for each voter. Quadratic voting, ${ }^{7}$ for example, allows a first glimpse of what may be possible in microdemocratic voting structures. Each voter in a given microdemocratic voting scenario may be allocated a maximum number of votes. Such votes may be allocated by priority to any issues the respective voter wants to engage with. The more important a given societal issue is for a given voter, the more votes the voter will allocate to the issue. For example, as more and more people become aware of the climate crisis faced by humanity, they will increasingly allocate their votes on issues that reflect their increasing awareness. Change thus becomes tangible as people allocate their votes prioritized by their then existing preferences and awareness. Moreover, part of the power of this microdemocratic approach derives from the higher levels of knowledge a given voter has on a particular issue that is evidenced by the voters' proportional allocation of votes on such issue. It is less likely that voters will allocate number one priority on issues they know relatively little about. Voting outcomes and their application and relevance to a given constituent are thus presumably improved.

Microdemocracies would need to address the problem of the tragedy of the commons. The concept of the tragedy of the commons describes the downsides of a microdemocratic shared-resource system for the common good of the society. In microdemocratic systems, individual voters act independent of the totality of voters according to their selfinterest. Acting for their own self-interest may be contrary to the common good of all voters. Without controls, some self-interested voters may be depleting or spoiling resources they share with the rest of society, such as the environment, public goods, among others. Similarly, majority rule may mean discrimination of the minority. A majority that is unaffected by a rule they instantiate may have a discriminatory impact on a minority that is disproportionately affected by the

\footnotetext{
${ }^{6}$ Wulf Kaal - December 20, 2019.

${ }^{7}$ Posner, Eric A., Weyl, E. Glen, Buterin, Vitalik, and Lanier, Jaron (2018). Radical Markets: Uprooting Capitalism and Democracy for a Just Society. Princeton University Press: Buterin, Vitalik, Hitzig, Zoe, N.J, \& Weyl, E. Glen (Dec. 2018). Liberal Radicalism: A Flexible Design for Philanthropic Matching Funds, https://papers.ssrn.com/sol3/papers.cfm?abstract_id=3243656 (last accessed June $1,2020)$
} 
change in the rules.

A key advantage of a microdemocracy is the speed of change it can initiate. In the early 2020s, representative democracies were largely slow and inflexible because of their constitutional and institutional infrastructure and their respective legal systems. Change typically depended on the election of a new government after, typically, around 4 years at the earliest. By contrast, microdemocratic voting would be a more immediate feedback system between voters and their representatives. Because technology enables the incorruptible counting and tallying of votes instantaneously, voting can be more dynamic and incremental and political will is exercised more directly.

Decentralized technologies provide inherent microdemocratic features that can help instantiate microdemocratic principles and processes in society. Decentralized technologies can help bring more direct microdemocratic representation to society. Decentralized technology enables smaller scale democratic decisions at unprecedented scale. In the early $21^{\text {st }}$ century, internet platform businesses and social media companies inaugurated new forms of voting. The "Like" button, while deeply-flawed in its incentive design and voting-related outcomes, inaugurated a new form of voting on social outcomes and, at the same time, trained the voting public to engage more directly with voting related outcomes. Decentralized technologies create extensions of these forms of more direct democratic votes. Unlike their centralized predecessors, decentralized technologies, for the first time in history, enable improved incentive designs that help overcome the insufficiencies in voting outcomes of representative democracies. ${ }^{8}$ As voting pools in decentralized systems increase, deepen, and proliferate, they can become supplemental voting systems that help overcome the lack of legitimacy and improve representative democracies. Of course, such upgrades to existing representative democracies may require constitutional or democratic support which is dubious at best.

Decentralized technology can improve voting technology in representative democracies. Voting technologies in existing representative democracies were woefully outdated at the beginning of the $21^{\text {st }}$ century. Since, Gore vs. Bush, ${ }^{9}$ which necessitated reexamination of punch holes in paper voting cards, existing voting systems with analog or paper technology have been proven suboptimal. But the problems with existing voting systems do not stop with technology. Redistricting, that is, changing the demarcation of a given voting district to adjust to voter preferences therein and change overall voting outcomes, and other basic democratic voting-related issues show that voting and the democratic institutions built to facilitate basic tenets of representative democracies have been under attack and require updates. Decentralized technologies have technological features that can provide solutions. But, such solutions also require political will. For example, voting on a public blockchain that overcomes the trilemma of blockchains (decentralization, scaling, security) may become a core application of the technology. Yet, governments in the existing representative democracies may not wish to surrender control over the voting process and may prefer to use private blockchains to facilitate voting outcomes. Decentralized technologies have the capacity to change the incentive design for voting which helps address some of the dangers presented by existing internet voting.

Centralized technology companies increasingly act like governments. For example, Microsoft announced in January 2020 that it would open a representation to the United Nations..$^{10}$ In 2020, executives of the top technology companies were added to list of attendees at the annual security conference, a position that was previously reserved exclusively for presidents, prime ministers, and politicians. Regulating the increasing power of centralized technology conglomerates only addresses part of the issues. Even if fully regulated, centralized technology conglomerates still have the power to set standards and norms for emerging technologies that government cannot effectively trace. Microdemocratic decision making helps set such standards outside of the control metrics imposed by the centralized technology conglomerates.

\section{Enhanced algorithmic future}

Decentralized systems enhance future algorithmic applications. Centralized algorithmic automation is a very powerful tool for humanity. It fulfills increasingly important roles in society for the betterment of humanity. It optimizes human interaction, outputs, and performance and can elevate most functions of technology-enhanced designs in society. At the same time, the conveniences and benefits that derive from centralized algorithmic automation bring with them risks to humanity that cannot be fully quantified. Decentralized systems can counteract the downsides and threats of algorithmic automation and thereby enhance the long-term benefits of technological solutions for humanity.

Centralized algorithmic automation is affecting society at a large scale. Since the early 2010 s, algorithmic functionalities and algorithms that utilize big data from sensors, IoT devices, and other big datasets, have started to take over significant

\footnotetext{
${ }^{8}$ For more details see Chapter 3 Future Decentralization.

${ }^{9}$ Bush vs. Gore, 531 U.S. 98 (2000).

${ }^{10}$ Schaake, Marietje. (Feb. 20, 2020). Big Tech Companies Want to Act Like Governments [Opinion]. Financial Times (FT.com).
} 
societal functions. As internet-based applications are becoming increasingly prevalent and used by humans, such as, Google, Facebook, and Amazon, such applications not only provide conveniences in humans' daily lives but also start accumulating more and more power through their functionalities in society. For example, a powerful application, such as Google has already a lot of control over human knowledge and thought. If it were to make disparate recommendations to similarly situated users, it could control societal outcomes by benefiting one user group with better predictions and outcomes over another user group.

Algorithmic functions can optimize bounded human rationality that has afflicted humanity since its inception. Human actions and associated outcomes are increasingly optimized through the availability of big data and algorithmic data analytics. For example, the increasing availability of health data and analytics lowers the likelihood of suboptimal human health-related choice, especially if reinforced with algorithmic applications that interact with humans. The quantification of human thought, feeling, and action and their algorithmic optimization can create optimization of humans that is too complex for humans to understand without the data-driven algorithmic aids. Health data through sensors, wearables etc. and sensors that observe human interaction with content on, for instance, a Kindle that scans health data while humans interact with text and other content, provide some context.

Given the analytical and predictive skills of algorithmic applications, data and algorithmic oracles can not just upgrade human action, experiences, and outputs, they can also upgrade otherwise human-generated collective societal functions. For example, algorithmic applications can incrementally upgrade democratic processes in society. Big data and algorithmic data analytics enable centralized algorithmically automated systems (such as, Google, Facebook, and Amazon in the early 2020s) to know individuals' political preferences better than the individual's themselves. ${ }^{11}$ Algorithmic data analytics-driven human choice removes humans' bounded rationality. ${ }^{12}$ As algorithmic analytics and predictions of human preferences and choice become increasingly accepted, exercising human political will by proxy through algorithmic interpretations may become increasingly the new normal in the foreseeable future.

Decentralized systems' ability to preserve the human element and coordinate human input enables the enhancements of algorithmic automation. In its ultimate state, algorithmic automation negates human input in data systems as humans' bounded rationality creates inefficiencies and the human condition results in suboptimal outputs, even when algorithmically optimized. Decentralized systems' interaction with and optimization of algorithmic societal functions overcomes centralized algorithmic automation' negative effects on equity, individualism, individual choice, free will, and diversity of human experiences, as we will further explain in Chapter 3 Future Decentralization. A feedback loop exists between decentralized protection of the human factor in algorithmic systems and the proliferation of both algorithmic automation and decentralized solutions.

Decentralized enhancements of algorithmic automation become more evident when comparing decentralized systems and centralized algorithmic automation. Key differences exist between the approaches and outcomes of decentralized systems and centralized algorithmic automation. Where centralized algorithmic automation would use centrally collected data analytics to enhance decision making metrics and associated outcomes, decentralized systems would make recommendations based on the wisdom of the crowds of humans, machines, and other entities combined. Where centralized algorithmic automation would systematically remove the human presence in an effort to optimize efficiency and outcomes, decentralized systems would use the power of algorithmic applications to enhance its features and outcomes with an omnipresent human backstop. Where centralized algorithmic automation would create an autocracy powered by centralized algorithmic human predictors, decentralized systems would create a meritocracy powered by optimized human decisions that increase diversity of choice for all. Where centralized algorithmic automation would create structures for the evolution of dataism, decentralized systems would attempt to remove the human condition, that is, remove the human shortcomings associated with bounded rationality and limited foresight that lead to suboptimal outcomes, with a view towards enhancing individualism for the benefit of humanity. Where centralized algorithmic automation would harmonize to enhance efficiency and associated outcomes, decentralized systems would emphasize diversity to improve outcomes. ${ }^{13}$

Decentralized systems are uniquely positioned to make recommendations for and improve human and societal functions that are algorithmically generated. Unlike centralized algorithmically enhanced systems that use centrally

\footnotetext{
${ }^{11}$ See further, Kahneman, Daniel, Fredrickson, Barbara L., Schreiber, Charles A., \& Redelmeier, Donald, A. (Nov. 1993). When More pain is preferred to less: Adding a better end. Psychological Science, 4(6), 401-405.

${ }^{12}$ Kahneman's cold water experiments suggest that humans listen to a narrating self in political decision making, follow a peak-end rule, forget the vast majority of political events during a given legislation period, focus exclusively on a few extreme outlier events, and give largely disproportionate weight in political decision making to the most recent events. Ibid.

${ }^{13}$ As Karl Popper put it: "The growth of knowledge depends entirely on disagreement", Popper, Karl, R. (1994). The myth of the framework: In defence of science and rationality. Routledge: London.
} 
collected data and analytics to enhance human decision-making metrics and associated outcomes, decentralized systems can use the combined wisdom of the crowds of humans, machines, and other entities. Decentralized systems can use uniquely diverse human inputs that over time can become automated in their applications but remains foundationally based on a human consensus that functions as a human backstop for automated algorithmic applications. For example, in the early 2020s, cryptocurrency startups have started to experiment with voting metrics, voting incentive designs, and voting outcomes. These evolving blockchain-based designs with human voting inputs can over time become automated. The basis of the evolving automated algorithmic designs remains a diverse human voting record. Because of its blockchain-based quasi-precedent nature with a human backstop, the decentralized system enables built-in checks and balances that can support resistance to even the most apocalyptic applications and takeover of algorithmic systems in human society that systematically attempt to remove the human presence in an effort to optimize efficiency and outcomes.

The enhancements offered by decentralized systems can be identified largely through the autocratic tendencies of algorithmic applications versus the microdemocratic tendencies of decentralized systems. Centralized algorithmic human predictors can be used by those few entities and individuals who control the data flow and the associated algorithms to create autocratic algorithmic systems and structures. The implied loss of democratic principles in society is perhaps an inevitable side effect. By contrast, decentralized systems thrive through diversity of human input and human experiences that enable smaller scale democratic decisions at unprecedented scale. Decentralized systems can further enhance their inherent microdemocratic processes and outputs through meritocratic principles that optimize incentive designs and human decision making. For example, at the beginning of the 2020s, blockchain startups experimented with censorproof, incorruptible, anonymous, and autonomous voting systems that required proof of voting pool validation of human inputs.

The enhancement of individualism is a key feature that distinguishes decentralized systems from centralized algorithmic automation and underscores its value proposition. Where centralized algorithmic automation would harmonize, decentralized systems would emphasize diversity for improved outcomes. Because of it autocratic and efficiency focused tendencies, centralized algorithmic automation is prone to create structures for the evolution of dataism, which ultimately denies the value of human inputs and individualism. By contrast, decentralized systems depend on and enhanced individualism. Decentralized systems thrive through human diversity. Unlike centralized algorithmic automation that ultimately attempts to remove the human inefficiencies in the system, decentralized systems create structures that enable incentive designs that help overcome human shortcomings associated with bounded rationality and limited foresight. These optimizations create a decentralized form of efficiency enhancement that reconciles with individualism for the benefit of humanity. For example, in the early 2020s, blockchain startups have started to experiment with tokenized incentive designs intended to overcome bounded rationality and limited foresight.

\section{Decentralized commerce}

Decentralized technology solutions create disruptions for existing legacy commerce. The emergence and proliferation of distributed blockchain applications (DApps) in the aftermath of the invention of the Bitcoin protocol in 2009 demonstrate that a nascent market for such applications and consumer demand already exists at the beginning of the 2020s. Consumer preferences continue to shape the DApps market and the solutions it may offer for commerce and society. A significant market for blockchain applications will be the corporate marketplace, banks, legal, realty, and insurance. These market will all be impacted very significantly. DApps create solutions for business and society that are subject to far fewer and rather different transaction costs compared with centralized legacy businesses in the same industries. Through codebased solutions, DApps can help increase the overall trust of consumers and market participants at an unprecedented scale. Despite many cybersecurity issues associated with cryptocurrencies, among others, code-based trust can help lower transaction costs, but it also increases consumer and overall market confidence and certainty, which facilitate economies of scale that are rarely possible in centralized structures. Through the lower cost structure, decentralized platforms have the ability to remove consumer fees that are an integral part of their centralized competitor businesses. Removing such centralized fees also allows for the removal of downward pressure on the platforms' worker compensation as costs are less likely to increase and be passed on to workers and consumers. The lack of fees can help create a more efficient marketplace through the removal of the rent seeking intermediators.

Decentralized technologies' ability to lower transaction costs by removing otherwise needed intermediaries disrupts existing business models that are based on intermediation. For example, in the early 2020s blockchain technology provided numerous examples of evolving decentralized technologies that create an independent and transparent platform for establishing truth and building trust. Intermediaries, bureaucracy and old-fashioned procedures are replaced by: code, connectivity, crowds, and collaboration. An example here is the Decentralized Finance (DeFi) market where increasingly financial products are offered on top of digital assets to facilitate disintermediation of legacy systems. The 
technology increases openness and speed, while at the same time significantly reducing costs by replacing intermediaries in, for example, traditional finance. But perhaps the most significant feature of blockchain technology is that it is so adaptable. The many applications of evolving blockchain technology have the potential to change business models. Because of its implicit code-based guarantees, the technology allows a qualitatively different solution for agency, a foundational element of capitalism. Blockchain technology can replace agency constructs for many business relationships. The guarantees ensure that participant cannot circumvent the rules embedded in the code, contracts execute only if and when all contract parameters were fulfilled by both parties and verified by a majority of miners/nodes in the system. Blockchain-based guarantees remove agency costs because principals are less required to institute oversight and monitoring of agents which addresses the inherent agency problems in modern finance and corporate governance.

Similarly, decentralized technologies reform business, administrative, and legal processes that rely on intermediaries. Any such processes may be updated for decentralized technologies. For example, corporate processes that have ledger functionality but rely on legal intermediaries could be streamlined very quickly by implementing blockchain technology. When blockchain technology becomes more widely accepted and applications spread into consumer-facing markets, legacy business processes and structures will likely be among the first to be amended. The combination of blockchain technology startups with platforms, AI, and machine learning present enormous opportunities to improve humanity. Especially if these technologies are used in combination with big data that is collected by using tech solutions and blockchain applications in combination with machine learning, it may be increasingly possible to create more creative and faster tools. This, in turn, creates a surge of new and innovative platforms with disruptive effects for many industries.

Decentralized payment systems' ability to rely entirely on cryptocurrencies creates comparative advantages over centralized systems. Centralized legacy fiat payment systems and platforms typically require some form of an existing banking relationship in order for consumers to utilize their services. Holding and storing cryptocurrencies does not require a banking relationship. While many legacy banks are cognizant of their limitations and are ready to embrace cryptocurrencies, centralized legacy fiat payment systems remain subject to payment processing issues and slow processing times for payments. They also require high fees for intermediaries that facilitate the payment process, such as, banks and PayPal, among others. The fees make it only economically viable for higher volumes of transactions, creating barriers to entry in the process. Decentralized payment systems are not subject to these limitations. Finally, while money laundering has traditionally been a problem in these markets, anonymity of market participants in cryptocurrency networks can also increase participation in certain markets and economies. As the technology and its uses evolve, traditional markets are increasingly encapsulated by it.

Legacy payment systems, cash, and bank notes are gradually losing ground to other payment systems. ${ }^{14}$ At the beginning of the 2020s, in Northern Europe, as few as one in every five transactions is made in cash. ${ }^{15}$ Cash usage in the United States, the United Kingdom, the Netherlands, Sweden, Finland, Canada, France, among other industrialized nations, has fallen well below $50 \%$ of total transaction volume. ${ }^{16}$ In the United States, transacting in cash costs the consumer around $\$ 200$ bn annually-about $\$ 637$ per person annually, which is a result of the cost of production, storage, and transportation, among other factors. ${ }^{17}$

The end of technological life cycles of legacy systems in the early 2020s and associated emerging trends in payment systems necessitate central banks' enhanced examination of alternative payment systems. ${ }^{18} \mathrm{Central}$

\footnotetext{
${ }^{14}$ Rogoff, Kenneth, S. (2016). The Curse of Cash. Princeton: Princeton University Press: Princeton; Brugge, Jonathan, Denecker, Olivier, Jawaid, Hamza, Kovacs, Andras., and Shami, Ibrahim. (Aug. 20, 2018). Attacking the Cost of Cash. McKinsey \& Company: Our Insights, https://www.mckinsey.com/industries/financial-services/our-insights/attacking-the-cost-of-cash (accessed June 1, 2020). 15 Ibid.

${ }^{16}$ Ibid.

${ }^{17}$ Ibid. The cost of cash is primarily associated with counting, managing, storing, transporting, guarding, and accounting for bank notes. Berensten, Aleksander., and Schar, Fabian. (2018). The case for Central Bank Electronic Money and the Non-case for Central Bank Cryptocurrencies. Federal Reserve Bank of St. Louis Review, 100(2), 97-106; The theft of cash alone costs U.S. retail businesses losses around $\$ 40$ bn annually. Yakowicz, Will (Sept. 20, 2013). Cash Costs U.S. Businesses \$40 bn a Year. Inc., https:// www.inc.com/will-yakowicz/dealing-with-cash-costs-american-businesses-55-billion.html (accessed June 1, 2020).

${ }_{18}$ Bech, Morten., and Garratt, Rodney. (2017). Central Bank Cryptocurrencies. BIS Quarterly Review.

${ }^{19}$ Hileman, Garrick., and Rauchs, Michel. (2017). Global Cryptocurrency Benchmarking Study. Cambridge Cenre for Alternative Finance, https:/www.jbs.cam.ac.uk/fileadmin/user_upload/research/centres/alternative-finance/downloads/2017-04-20-globalcryptocurrency-benchmarking-study.pdf (accessed June 1, 2020); Mersch, Yves. (July 24, 2017). Central Bank Speech at the Cent. Bank of Malaysia Monetary Policy Conference [transcript available at https://www.bis.org/review/r170807c.htm]; Digital Currency Initiative, MIT Media Lab, https://dci.mit.edu; Monetary Authority of Singapore, Financial Stability Review (Nov. 2017), https:// www.mas.gov.sg/-/media/MAS/resource/publications/fsr/FSR-2017.pdf; Meaning, Jack, Dyson, Ben, Barker, James., and Clayton, Emily (May 2018). Broadening Narrow Money: Monetary Policy with a Central Bank Digital Currency. Bank of England [Staff Working Paper No. 724]; Koning, J.P. (2016). Fedcoin: A Central Bank-Issued Cryptocurrency, R3 Report, 15; Motamedi, Sina. (July 21, 2014). Will Bitcoins Ever Become Money? A Path to Decentralized Central Banking, TannuTuva.org, https://tannutuva.org/ 2014/will-bitcoins-ever-become-money-a-path-to-decentralized-central-banking
} 
banks and governments around the world have been experimenting with government-sponsored digital and cryptocurrencies since 2015. ${ }^{19}$ In the case of central banks, such experimentation was already close to launch ${ }^{20}$ or fully operational in the early 2020s. ${ }^{21}$ Most major tech companies in the private sector have been experimenting with cryptocurrency projects since 2017. Several governments have issued their own digital currencies. Examples include Tunisia (eDinar), ${ }^{22}$ Senegal (eCFA), ${ }^{23}$ Sweden (eKrona), ${ }^{24}$ Dubai (EmCash), ${ }^{25}$ Japan (Jcoin), ${ }^{26}$ Estonia (Estcoin) ${ }^{27}$ and Ecuador, ${ }^{28}$ among others. ${ }^{29}$

\section{References}

Bech, Morten., and Garratt, Rodney. (2017). Central Bank Cryptocurrencies. BIS Quarterly Review.

Berensten, Aleksander., and Schar, Fabian. (2018). The case for central bank electronic money and the non-case for central bank cryptocurrencies. Federal Reserve Bank of St. Louis Review, 100(2), 97-106.

Billner, Amanda (Oct. 26, 2018). Now There are Plans for 'e-Krona' in Cash-Shy Sweden. Bloomberg: Economics, https:/ /www.bloomberg.com/news/articles/2018-10-26/riksbank-to-develop-pilot-electronic-currency-amid-cash-decline.

Browne, Ryan (Feb. 20, 2018). Venezuela About to Pre-Sell 'Petro' Cryptocurrency, and Other Countries Could Follow. CNBC.com, https://www.cnbc.com/2018/02/19/venezuela-petro-cryptocurrency-pre-sale-starts-february-20.html.

Brugge, Jonathan, Denecker, Olivier, Jawaid, Hamza, Kovacs, Andras., and Shami, Ibrahim. (Aug. 20, 2018). Attacking the Cost of Cash. McKinsey \& Company: Our Insights, https://www.mckinsey.com/industries/financial-services/ our-insights/attacking-the-cost-of-cash (accessed June 1, 2020).

Buck, Jon (Oct. 1, 2017). Dubai will issue first ever state cryptocurrency. Cointelegraph, https://cointelegraph.com/ news/dubai-will-issue-first-ever-state-cryptocurrency.

Bush vs. Gore, 531 U.S. 98 (2000).

Buterin, Vitalik, Hitzig, Zoe., and Weyl, E. Glen. (Dec. 2018). Liberal Radicalism: A Flexible Design for Philanthropic Matching Funds [unpublished article], https://papers.ssrn.com/sol3/papers.cfm?abstract_id=3243656 (accessed June 1, 2020).

Chohan, Usman, W. (Feb. 7, 2018). Cryptocurrencies as Asset-Backed Instruments: The Venezuelan Petro [unpublished article], https://papers.ssrn.com/sol3/papers.cfm?abstract_id=3119606.

Das, Samburaj. (Nov. 28, 2016). Senegal Will Introduce a Blockchain-Based National Digital Currency. CCN, https:// www.ccn.com/senegal-will-introduce-blockchain-based-national-digital-currency/.

Digital Currency Initiative, MIT Media Lab, https://dci.mit.edu;

${ }^{20}$ The Bank of Canada and Bank of England announced in 2016 that the technology was not ready for a central bank-sponsored cryptocurrency. Yet other central banks, such as in Singapore and Sweden, have already launched their e-currency projects. See Chapter 4, footnote 18 .

${ }^{21}$ Chohan, Usman, W. (Feb. 7, 2018). Cryptocurrencies as Asset-Backed Instruments: The Venezuelan Petro [unpublished paper], https://papers.ssrn.com/sol3/papers.cfm?abstract_id=3119606; Zagaris, Bruce (2018). U.S. Bans Venezuela's New Cryptocurrency and adds 3 officials to sanctions list. International Enforcement Law Reporter, 34:3, 157-161; Hirschfeld Davis, Julie., and Popper, Nathaniel. (Mar. 20, 2018). White House Bans Venezuela's digital currency and imposes further sanctions. New York Times, A1.

${ }^{22}$ Kastelein, Richard. (Dec. 28, 2015). Tunisia to Replace Its National Digital Currency. eDinar. with Blockchain-Driven Monetas Currency. Blockchain News, https:/www.the-blockchain.com/2015/12/28/tunisia-to-replace-its-national-digital-currency-edinar-withblockchain-driven-monetas-currency/ (accessed June 1, 2020).

${ }^{23}$ Das, Samburaj. (Nov. 28, 2016). Senegal Will Introduce a Blockchain-Based National Digital Currency. CCN, https://www.ccn.com/ senegal-will-introduce-blockchain-based-national-digital-currency/.

${ }^{24}$ Afraid of a few commercial entities controlling cash supply in Sweden, the Swedish central bank has kicked off its digital currency project e-krona. Billner, Amanda. (Oct. 26, 2018). Now There are Plans for 'e-Krona' in Cash-Shy Sweden. Bloomberg: Economics, https://www.bloomberg.com/news/articles/2018-10-26/riksbank-to-develop-pilot-electronic-currency-amid-cash-decline.

${ }^{25}$ Buck, Jon. (Oct. 1, 2017). Dubai will issue first ever state cryptocurrency. Cointelegraph, https://cointelegraph.com/news/dubaiwill-issue-first-ever-state-cryptocurrency.

${ }^{26}$ Kharpal, Arjun. (Sep. 27, 2017). Japanese banks are thinking of making their own cryptocurrency called the J-Coin. CNBC: Tech Transformers, https://www.cnbc.com/2017/09/27/japanese-banks-cryptocurrency-j-coin.html.

${ }^{27}$ Korjus, Kaspar. (Dec. 18, 2017). We're planning to launch estcoin - and that's only the start. Medium, https://medium.com/eresidency-blog/were-planning-to-launch-estcoin-and-that-s-only-the-start-310aba7f3790.

${ }^{28}$ Rosenfeld, Everett. (Feb. 9, 2015). Exuador becomes the first country to roll out its own digital cash. CNBC.com, https:// www.cnbc.com/2015/02/06/ecuador-becomes-the-first-country-to-roll-out-its-own-digital-durrency.html.

29 Thomson, Reuters. (Oct. 25, 2017). Cryptocurrencies by Country, https://blogs.thomsonreuters.com/answerson/worldcryptocurrencies-country/ 
Hileman, Garrick., and Rauchs, Michel. (2017). Global Cryptocurrency Benchmarking Study. Cambridge Centre for Alternative Finance, https://www.jbs.cam.ac.uk/fileadmin/user_upload/research/centres/alternative-finance/ downloads/2017-04-20-global-cryptocurrency-benchmarking-study.pdf (accessed June 1, 2020)

Hirschfeld Davis, Julie., and Popper, Nathaniel. (Mar. 20, 2018). White house bans venezuela's digital currency and imposes further sanctions. New York Times, A1.

Kahneman, Daniel, Fredrickson, Barbara L., Schreiber, Charles A., and Redelmeier, Donald A. (Nov. 1993). When more pain is preferred to less: Adding a better end. Psychological Science, 4(6), 401-405.

Kant, Immanuel. (1785). Grundlegung zur Metaphysik der Sitten. Riga, J.F. Hartknoch.

Kastelein, Richard. (Dec. 28, 2015). Tunisia to replace its national digital currency, edinar, with blockchain-driven monetas currency. Blockchain News, https://www.the-blockchain.com/2015/12/28/tunisia-to-replace-its-national-digitalcurrency-edinar-with-blockchain-driven-monetas-currency/ (accessed June 1, 2020).

Kharpal, Arjun. (Sep. 27, 2017). Japanese banks are thinking of making their own cryptocurrency called the j-coin. CNBC: Tech Transformers, https://www.cnbc.com/2017/09/27/japanese-banks-cryptocurrency-j-coin.html.

Koning, J.P. (2016). Fedcoin: A Central Bank-Issued Cryptocurrency, R3 Report, 15

Korjus, Kaspar (Dec. 18, 2017). We're Planning to Launch estcoin - and That's Only the Start. Medium, https:// medium.com/e-residency-blog/were-planning-to-launch-estcoin-and-that-s-only-the-start-310aba7f3790.

Meaning, Jack., Dyson, Ben, Barker, James., and Clayton, Emily. (May 2018). Broadening Narrow Money: Monetary Policy with a Central Bank Digital Currency. Bank of England [Staff Working Paper No. 724]

Mersch, Yves. (July 24, 2017). Central Bank Speech at the Cent. Bank of Malaysia Monetary Policy Conference [transcript available at https://www.bis.org/review/r170807c.htm]

Monetary Authority of Singapore, Financial Stability Review (Nov. 2017), https://www.mas.gov.sg/-/media/MAS/resource/ publications/fsr/FSR-2017.pdf

Motamedi, Sina. (July 21, 2014). Will bitcoins ever become money? A path to decentralized central banking, TannuTuva.org, https://tannutuva.org/2014/will-bitcoins-ever-become-money-a-path-to-decentralized-central-banking.

Popper, Karl R. (1994). The Myth of the Framework: In Defense of Science and Rationality. Routledge: London.

Posner, Eric A., Weyl, E. Glen, Buterin, Vitalik, and Lanier, Jaron (2018). Radical Markets: Uprooting Capitalism and Democracy for a Just Society. Princeton University Press: N.J.

Rogoff, Kenneth S. (2016). The Curse of Cash. Princeton: Princeton University Press: Princeton.

Rosenfeld, Everett (Feb. 9, 2015). Exuador becomes the first country to roll out its own digital cash. CNBC.com, https:/ /www.cnbc.com/2015/02/06/ecuador-becomes-the-first-country-to-roll-out-its-own-digital-durrency.html.

Schaake, Marietje (Feb. 20, 2020). Big Tech Companies Want to Act Like Governments [Opinion]. Financial Times (FT.com).

Thomson Reuters (Oct. 25, 2017). Cryptocurrencies by Country, https://blogs.thomsonreuters.com/answerson/worldcryptocurrencies-country/.

Wikipedia, Collectivism, https://en.wikipedia.org/wiki/Collectivism (accessed June 1, 2020).

Wikipedia, Individualism, https://en.wikipedia.org/wiki/Individualism (accessed June 1, 2020).

Yakowicz, Will (Sept. 20, 2013). Cash Costs U.S. Businesses $\$ 40$ Billion a Year. Inc.. https://www.inc.com/will-yakowicz/ dealing-with-cash-costs-american-businesses-55-billion.html (accessed June 1, 2020).

Zagaris, Bruce (2018). U.S. Bans Venezuela's New Cryptocurrency and Adds 3 Officials to Sanctions List. International Enforcement Law Reporter, 34(3), 157-161.

Cite this article as: Wulf A. Kaal (2021). H ow decentral ized systems can upgrade A I. I nternational J ournal of A rtificial Intelligenceand M achineLearning, 1(1), 1-10. doi: 10.51483/ IJAIM L.1.1.2021.1-10. 